\title{
Clinical Manifestations of Adult-Onset Still's Disease
}

\author{
Cihan Semet ${ }^{\mathbf{1}}$ (D), Ayşe Sena Şişman¹, Tuğba Çınar ${ }^{\mathbf{1}}$ (D), İsmail Anaç² (D), Yavuz Pehlivan (iD, \\ Halis Akalın ${ }^{\mathbf{1}}$ iD \\ 1 Department of Infectious Diseases and Clin Microbiology, Uludag University School of Medicine, Bursa, Turkey \\ 2 Department of Internal Medicine, Uludag University School of Medicine, Bursa, Turkey \\ 3 Department of Rheumatology, Uludag University School of Medicine, Bursa, Turkey
}

1 8-year-old male patient presented to our emergency room (ER) with complaints of swelling and temperature increase in the right wrist, pain in the right knee, and a rash without any itchiness on the palms and soles continuing for a week. He had no known systemic disease or any regular medication use. He had a history of a tick bite three months ago. The patient had neither any pet contact nor a fresh milk and cheese consumption history. Detailed anamnesis revealed that he had a sore throat a week prior to the present complaints. His rash started on the dorsal hand, and his fever was accompanied by a rash on the trunk and upper limbs in the following days. The patient had previously visited the ER with the current complaints, and amoxicillin/ clavulanate was prescribed with a pre-diagnosis of pharyngitis. Physical examination on admission revealed a body temperature of $37.5^{\circ} \mathrm{C}$, a pulse rate of $100 /$ minute, and an arterial blood pressure of 120/60 $\mathrm{mmHg}$. There were macular rashes on his palms and soles, swelling and temperature increase on the right-hand metacarpal joint, and increased pain with motion in the right knee joint. There was no splenomegaly or lymphadenopathy. Laboratory findings were as follows: leukocyte, 22.0 x 103/uL (neutrophil 83\%, lymphocyte 10\%, monocyte 6\%, and basophil 1\%); Hgb, $12.3 \mathrm{gr} / \mathrm{dL}$; platelet, $251 \times$ 103/uL; AST, 33 U/L; ALT, 26 U/L; erythrocyte sedimentation rate, 42 mm/hour; and CRP, $33.3 \mathrm{mg} / \mathrm{dL}$. During follow-up, the patient developed a $38.7^{\circ} \mathrm{C}$ fever accompanied by rash on the trunk, neck, and upper extremity (Figure 1). His ferritin level was 8,871 ng/mL; ASO, $44 \mathrm{IU} / \mathrm{mL}$; RF, < $2 \mathrm{IU} / \mathrm{mL}$; Anti CCP, < $0.5 \mathrm{U} / \mathrm{mL}$; and ANA was negative. Lyme IgM and IgG (ELISA) were negative. Anti-HIV, CMV IgM, and EBV-VCA IgM were negative. CMV IgG and EBV-VCA IgG were positive. No effusion was observed on the ultrasonography of the right knee joint. Abdominopelvic ultrasonography and transthoracic echocardiography were normal. There was no growth in the blood and urine cultures. The patient was diagnosed as Adult-Onset Still's Disease by rheumatology and given 1,000 mg/day of prednisolone for three days. The patient's complaints regressed on the 4 th day of treatment, and he was discharged with prescriptions for 40 $\mathrm{mg}$ of prednisolone and methotrexate tablets of $12.5 \mathrm{mg} / \mathrm{week}$.

Corresponding Author: Halis Akalın

E-mail: halis@uludag.edu.tr

Received: June 9, 2020 Accepted: August 19, 2020 Published: August 31, 2020

\section{Suggested citation:}

Semet C, Sena Şişman A, Çınar T, Anaç İ, Pehlivan Y, Akalın $\mathrm{H}$. Clinical Manifestations of AdultOnset Still's Disease. Infect Dis Clin Microbiol 2020; 2: 106-107.

DOI: $10.36519 / \mathrm{idcm} .2020 .0015$ 


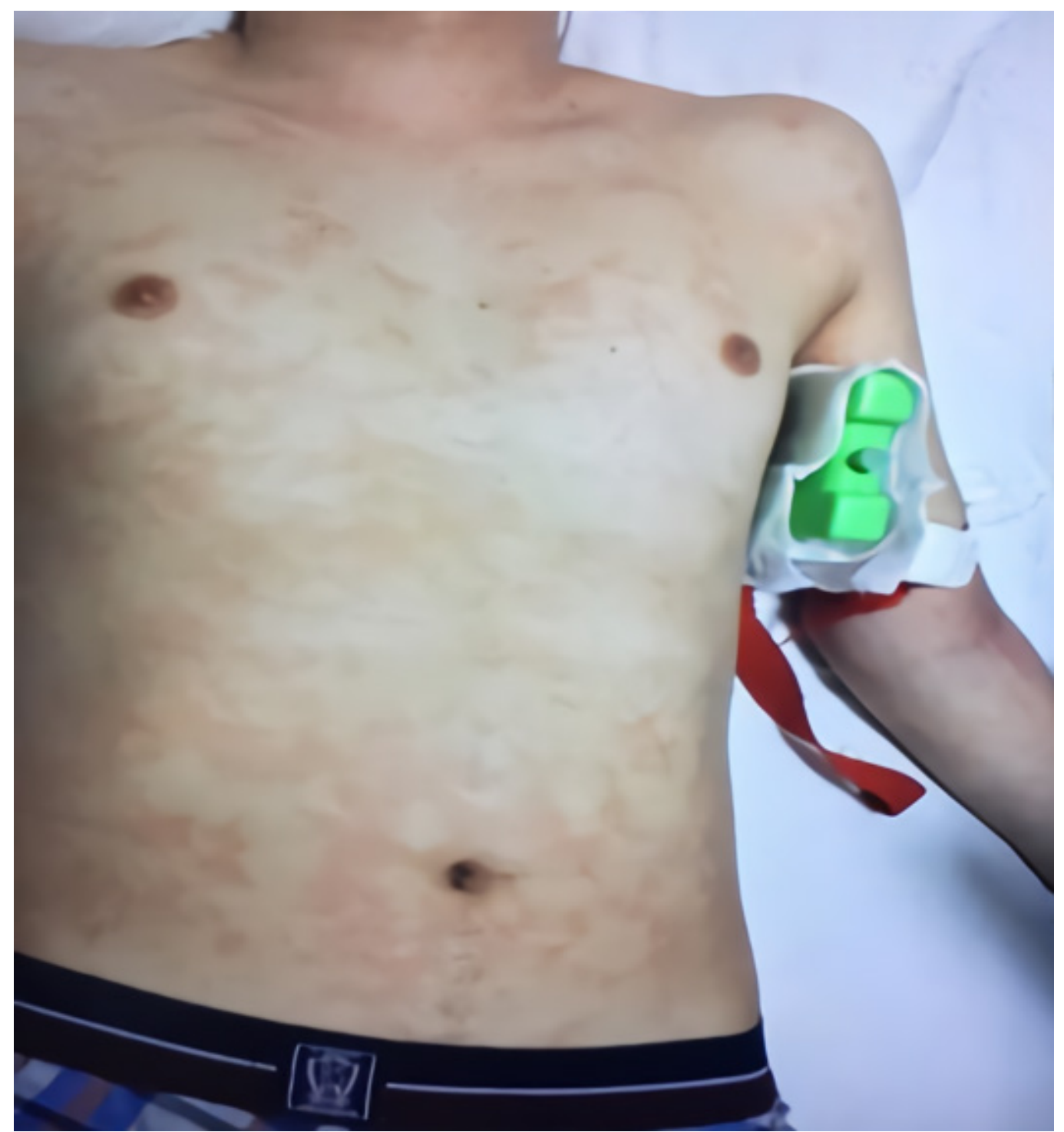

Figure 1.

Peer-review: Externally peer-reviewed

Conflict of Interest: The authors have no conflict of interest to declare.
Financial Disclosure: The authors declared that this study has received no financial support. 